\title{
Neovascularization Failure of an Interposed Free Intestinal Colon Flap 10 Years after Esophageal Reconstruction
}

\author{
Oscar J. Manrique, MD ${ }^{1}$ Shih-Heng Chen, $\mathrm{MD}^{2}$ Pedro Ciudad, MD, PhD ${ }^{3}$ Hung-Chi Chen, MD, PhD ${ }^{3}$
}

${ }^{1}$ Division of Plastic and Reconstructive Surgery, Mayo Clinic, Rochester, Minnesota

2 Department of Plastic and Reconstructive Surgery, China Medical University Hospital, Taichung, Taiwan

${ }^{3}$ Department of Plastic and Reconstructive Surgery, Chang Gung Memorial Hospital, Linkou, Taiwan

J Reconstr Microsurg Open 2017;2:e35-e36.

After free tissue transfer, the recipient bed plays an important role in the neovascularization process by maintaining flap viability independent of its primary vascular pedicle. ${ }^{1}$ However, there is still some controversy of how long the patency of the primary vascular pedicle must be maintained to ensure free flap survival. ${ }^{2}$ Factors such as the type of flap,
Address for correspondence Oscar J. Manrique, MD, Division of Plastic and Reconstructive Surgery, Mayo Clinic, 200 First Street SW. Rochester, MN 55905 (e-mail: manrique.oscar@mayo.edu).

prior radiation, and active recipient site infection have shown to delay this neovascularization process. ${ }^{3,4}$

We report the case of a 58-year-old woman, with esophageal injury after alkali ingestion that was reconstructed with a free bowel conduit. Following complete assessment, she underwent total esophagectomy and feeding jejunostomy. After

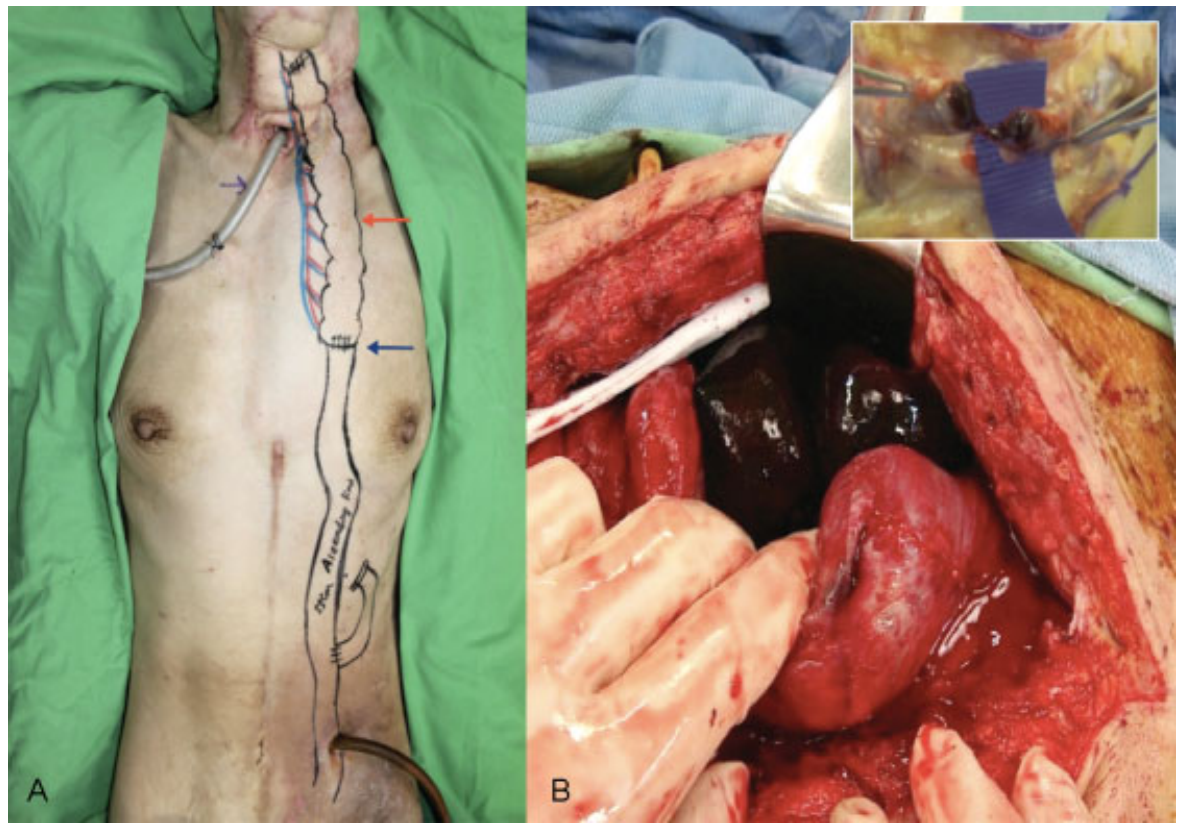

Fig. 1 (A) Schematic representation of the surgical procedure. A free colon conduit, tunneled under the subcutaneous tissue of the left chest. Free bowel conduit (red arrow) anastomosed to the Roux-Y Jejunum (blue arrow) with a distal jejunostomy for enteral nutrition. (B) Surgical exploration shows necrosis of the free bowel conduit. Right upper panel shows thrombosis of the vascular pedicle.

received

September 19, 2016 accepted after revision March 6, 2017
DOI http://dx.doi.org/ $10.1055 / \mathrm{s}-0037-1601850$. ISSN 2377-0813.
Copyright $\odot 2017$ by Thieme Medical Publishers, Inc., 333 Seventh Avenue, New York, NY 10001, USA Tel: +1(212) 584-4662.
License terms

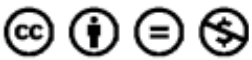


adequate nutritional support, the esophagus was reconstructed with a free transverse colon flap. During arterial microanastomosis mild arteriosclerosis was found; however, it was performed successfully.

The transferred colon segment was placed in a subcutaneous tunnel under the left chest, to assess conduit viability and improve its neovascularization by direct contact of the flap with the subcutaneous tissue (-Fig. 1A).

Three weeks after surgery, a Gastrografin swallow confirmed no leaks, strictures, or other complications, and diet was advanced. During further follow-up, the patient did not complain of dysphagia and was tolerating a regular diet.

However, 10 years later, she presented to another hospital with acute swelling, erythema, and pain over the skin covering the colonic conduit. She was admitted to the hospital for observation and managed conservatively.

After a couple of days, her condition deteriorated and the patient was then transferred to our hospital for further assessment. Upon arrival, she presented with worsening erythema and tenderness over the conduit site and was taken immediately to the operating room. Exploration was performed finding a necrotic bowel conduit with thrombosis of its vascular pedicle (-Fig. 1B). The flap was removed; however, her condition did not improve and she developed a systemic inflammatory response syndrome dying the following day.

Human and animal models have described the neovascularization process after free tissue transfer. There are many theories about what could accelerate or delay this process and what would be the appropriate time for a free flap to survive independently from its main pedicle. ${ }^{5}$

The serosa works as a natural bowel layer, preventing adhesion formation and promoting fluid secretion, thus minimizing friction. Because of its connective tissue properties, we believe that this layer can also play an important role in delaying or preventing the neovascularization of serosal conduits at its new recipient site.
In free flap surgery, there are two sources of blood supply, based on its own transferred pedicle and/or from surrounding structures (neovascularization).

Apparently in this case, the flap was supplied only by its main pedicle, because during intraoperative findings (-Fig. 1), we did not see any tissue connections between the transferred flap and the adjacent structures.

Based on our current studies (unpublished-data) in animal models of ischemia/reperfusion comparing free bowel conduits with and without serosa, we have seen strong similarities. However, further studies are necessary to support these clinical findings.

Note

None of the authors has a financial interest in any of the products, devices, or drugs mentioned in this manuscript.

Funding

None.

\section{References}

1 Wise SR, Harsha WJ, Kim N, Hayden RE. Free flap survival despite early loss of the vascular pedicle. Head Neck 2011;33(07): 1068-1071

2 Burns A, Avery BS, Edge CJ. Survival of microvascular free flaps in head and neck surgery after early interruption of the vascular pedicle. Br J Oral Maxillofac Surg 2005;43(05):426-427

3 Mücke T, Wolff KD, Rau A, Kehl V, Mitchell DA, Steiner T. Autonomization of free flaps in the oral cavity: a prospective clinical study. Microsurgery 2012;32(03):201-206

4 Weinzweig N, Gonzalez M. Free tissue failure is not an all-or-none phenomenon. Plast Reconstr Surg 1995;96(03):648-660

5 Granzow J, Li AI, Caton A, Boyd JB. Free flap survival following failure of the vascular pedicle. Ann Plast Surg 2015;75(01):44-48 